\title{
Molecular Features and Methylation Status in Early Onset ( $\leq 40$ Years) Colorectal Cancer: A Population Based, Case-Control Study
}

\author{
Giulia Magnani, ${ }^{1}$ Daniela Furlan, ${ }^{2}$ Nora Sahnane, ${ }^{2}$ \\ Luca Reggiani Bonetti, ${ }^{3}$ Federica Domati, ${ }^{1}$ and Monica Pedroni ${ }^{1}$ \\ ${ }^{1}$ Dipartimento di Medicina Diagnostica, Clinica e Sanità Pubblica, Università di Modena e Reggio Emilia, 41124 Modena, Italy \\ ${ }^{2}$ Dipartimento di Chirurgia e Scienze Morfologiche, Università dell'Insubria, 21100 Varese, Italy \\ ${ }^{3}$ Divisione di Anatomia Patologica, Policlinico di Modena, 41124 Modena, Italy \\ Correspondence should be addressed to Monica Pedroni; monica.pedroni@unimore.it
}

Received 20 January 2015; Accepted 2 April 2015

Academic Editor: Miroslav Zavoral

Copyright (C) 2015 Giulia Magnani et al. This is an open access article distributed under the Creative Commons Attribution License, which permits unrestricted use, distribution, and reproduction in any medium, provided the original work is properly cited.

Colorectal cancer is usually considered a disease of the elderly. However, a small fraction of patients develops colorectal cancer earlier. The aim of our study was to define the frequency of known hereditary colorectal syndromes and to characterise genetic and epigenetic features of early nonhereditary tumors. Thirty-three patients $\leq 40$ years with diagnosis of colorectal cancer and 41 patients with disease at $>60$ years of age were investigated for MSI, Mismatch Repair proteins expression, KRAS and $B R A F$ mutations, hypermethylation, and LINE-1 hypomethylation. Detection of germline mutations was performed in Mismatch Repair, APC and MUTYH genes. Early onset colorectal cancer showed a high incidence of hereditary forms (18\%). KRAS mutations were detected in $36 \%$ of early nonhereditary tumors. Early onset colorectal cancer disclosed an average number of methylated genes significantly lower when compared to the controls $(p=0.02)$. Finally both of the two groups were highly methylated in ESR1, GATA5, and WT1 genes and were similar for LINE-1 hypomethylation. The genetic make-up of carcinomas differs from young to elderly patients. Early onset tumors showed more frequently a constitutional defective of Mismatch Repair System and a minor number of methylated genes. Hypermethylation of ESR1, GATA5, and WT1 genes suggests possible markers in the earlier diagnosis of colorectal tumorigenesis.

\section{Introduction}

Colorectal cancer (CRC) is the second most common cancer in both men and women in Western Europe, North America, Australia/New Zealand, and Japan, whereas it remains less frequent in Africa and Asia [1]. Colorectal cancer is usually considered a disease of the elderly in both sexes; data from Cancer Registries indicate that age-specific cancer incidence rises sharply after the age of 50-55 years and that mean age of affected individuals is around 70 years [2]. However, in a small fraction of patients (2-3\% of all affected individuals), colorectal malignancies may develop earlier [3].

A review of the Surveillance Epidemiology and End Results (SEER) Program data from 2005 to 2009 provides more detailed information regarding colorectal cancer in younger patients suggesting that the incidence in younger patients is increasing along time (ages $<20$ to 54 years) [4], whereas other data would show a relative stability of the rates [5]. Part of these discrepancies can be attributed to the different age-limits proposed for the definition of early onset (or "juvenile") tumors, which have been set at age 40,45 , or 50 depending on the authors and purposes of the studies $[6,7]$. Although there is no clear age cutoff defined, the majority of studies classify patients diagnosed with cancer at age $<40$ years as "early onset" (or "young") [8]. The reasons whereby some individuals develop colorectal cancer at an unusual age are poorly understood. Therefore, the etiology and biological characterisation of the majority of early onset colorectal neoplasms remain poorly defined. 
From a molecular point of view, early onset tumors represent a heterogeneous group of diseases, including known hereditary syndromes, familial cases, and apparently sporadic colorectal cancer. Hereditary diseases explain only part of early onset colorectal cancer [9] and mainly include Lynch syndrome [7, 10-12], Familial Adenomatous Polyposis (FAP) [13], Peutz-Jeghers syndrome [14], Cowden syndrome/Bannayan [15], and MUTYH-associated polyposis (MAP) $[13,16]$.

Recent studies have suggested that the clinic-pathologic features related to early onset colorectal cancer [7] are more likely to be present at advanced stages, to be poorly differentiated, and to be located in the distal colon and in the rectum, although marked differences have never been reported. Morphologically, early onset CRCs more frequently display adverse histological features, such as signet ring cell differentiation and perineural and venous invasion [9]. Investigation of somatic profiles showed an increasing number of tumors with KRAS, BRAF, and PIK3CA oncogenes mutations related to advanced age. Mutations in the tumor suppressor genes TP53 and PTEN were more frequent in the early onset group [17]. Other findings showed that young patients had significantly more chromosomal aberrations in their tumors than patients aged $>70$ years and microsatellite stable (MSS) colorectal cancer [7, 17, 18]. Recently, LINE-1 hypomethylation has been reported to be a distinct feature of MSS young age colorectal cancers ( $\leq 45$ years), suggesting that the genomic hypomethylation may represent a possible pathway of early onset colorectal carcinogenesis [19]. By contrast, very few data are available about the frequency of CpG island methylator phenotype (CIMP) in early onset CRCs [19].

The knowledge about this clinically and genetically heterogeneous group of colorectal cancers remains limited and further studies are essential. To address this issue, we conducted a population based case-control study on CRCs diagnosed before the age of 40 years in order to define the frequency of known hereditary CRC syndromes in this subset of patients and to better characterize genetic and epigenetic features of early nonhereditary tumors compared with sporadic late onset CRCs.

\section{Materials and Methods}

2.1. Patients. The patients described in this study were recruited through the specialized Colorectal Cancer Registry of Modena in the period 1984-2008. In the 25 years of registration, we recruited 38 patients affected by adenocarcinoma in the colon-rectum diagnosed $\leq 40$ years, which represented about $1 \%$ of the overall adenocarcinomas diagnosed (4,692 cases). We collected the biological material (blood sample, normal colorectal mucosa, and tumoral tissue) of 33 cases out of 38 and investigated the family history relative to the cases, including at least first-degree or second-degree relatives.

In order to compare some molecular features-including methylation of DNA - of early onset colorectal cancer with maturity onset disease, we selected a specific control group composed of 41 patients with late onset colorectal cancer (age-range: 61-85 years) that were matched to the study group for the following characteristics: sex, tumor location, and stage. Family cancer history was not present in late onset group. Tumors were classified as low-grade and highgrade adenocarcinoma [20] and staged with the Dukes' classification.

The study was approved by the local ethics committee. All individuals, or first-degree relative in case of death of the index case, gave their written consent for blood samples and tissue specimen analysis.

2.2. DNA Extraction. Genomic DNA from each patient was extracted by formalin-fixed, paraffin-embedded normal and tumor tissues. Serial sections from paraffin-embedded matched normal and neoplastic primary tissue were stained with Hematoxylin-eosin; representative normal and tumor regions were identified by microscopic examination. Areas of tumor tissue with more than $80 \%$ of malignant cells were selected in all cases, as previously described in detail [21]. Constitutional DNA from peripheral blood was obtained using QIAamp DNA Mini kit (Qiagen), according to the manufacturer's instructions.

2.3. MSI Analysis. MSI status of all tumors was evaluated using four fluorescent-labeled mononucleotide markers: BAT25, BAT26, NR24, and CAT25. These quasimonomorphic markers were selected after in-depth review of the literature for their very high sensitivity and specificity in identifying Mismatch Repair-deficient tumors [22-24]. The resulting panel composed of four markers reduced the time and cost involved in MSI testing. Other authors as Xicola and Deschoolmeester suggested a MSI analysis shortcut [23, 24]. Using this mononucleotide markers panel, a tumor was defined as MSI(+) when showing instability with at least three markers. MSI analysis was performed as described previously [21].

2.4. Immunohistochemical Analysis of MMR Proteins. Immunohistochemical (IHC) evaluation of MMR proteins expression was carried out on paraffin-embedded tissue sections of all tumors. The following mouse monoclonal antibodies were used: anti-MLH1, anti-MSH2 (PharMingen, San Diego, CA), and anti-MSH6 Transduction Laboratories, BD Biosciences, Brazil. For PMS2 protein, a rabbit monoclonal antibody was used (Ventana Roche Diagnostic, Italy). Immunostaining was executed by the avidin-biotin peroxidase technique; diaminobenzidine was used as a chromogen. Staining was carried out in NEX-ES Automatic Staining System, after counterstaining with Hematoxylin. Normal tissue and stromal cells or lymphocytes adjacent to the respective tumor were used as internal positive controls. Loss of MMR proteins expression was defined as complete absence of nuclear staining in tumor cells (but maintained in normal epithelial and stromal cells).

2.5. Analysis of Germline Mutations in MMR, APC, and MUTYH Genes. Cases showing MSI and lack of expression of one of MMR proteins in tumors were investigated to detect germline mutations in $M M R$ genes. Patients with clinical 
features of Familial Adenomatous Polyposis were assessed for $A P C$ gene and all young patients were screened for the presence of MUTYH gene mutations. Analysis of germline mutations was performed by direct sequencing of the PCR products obtained using the Dye Terminator Cycle Sequencing Kit (CEQ DTCS Kit, Beckman Coulter) and reactions were run on a CEQ 8000 capillary sequencer (Beckman Coulter). To exclude the possibility of large genomic rearrangements in $M M R$ genes, we used Multiplex Ligation-Dependent Probe Amplification (MLPA) procedure by SALSA P003-B2 kit (MRC-Holland, Amsterdam, Netherlands) and to confirm results SALSA P248-A2 kit (MRC-Holland). The entire open reading frame of $A P C$ gene was also analysed for the presence of deletions or rearrangements by using the SALSA P043 kit (MRC-Holland). Pathogenic mutations were detected twice and confirmed in a second blood sample of the patient.

2.6. Somatic BRAF and KRAS Mutations Analysis. All 74 tumors were analysed for $K R A S$ and $B R A F$ activating mutations. In KRAS, the more frequently mutated codons 12, 13 , and 61 were analysed [25]. In BRAF gene, we amplified exon 15 which includes the hot spot for mutation codon 600 (V600E). Mutation analysis was performed by direct sequencing with the use of the standard protocol and running on Beckman Coulter CEQ 8000 instrument.

2.7. Methylation-Specific Multiplex Ligation-Dependent Probe Amplification (MS-MLPA) Analysis. MS-MLPA analysis was performed on all the 74 tumors (33 early onset CRC and 41 control cases) using the ME001 MS-MLPA Tumor Suppressor-1 Kit, the ME002 MS-MLPA Tumor Suppressor2 Kit, and the ME011 MS-MLPA Mismatch Repair Genes Probemix Kit (MRC-Holland, Amsterdam, Netherlands). Using these three kits, a total of 38 tumor suppressor genes were analysed for aberrant promoter methylation. All these genes are frequently silenced by methylation in tumors of different sites, and they frequently harbour genetic alterations during tumorigenesis. Methylation-specific MLPA (MS-MLPA) is a semiquantitative method for methylation profiling. MS-MLPA is a variant of the MLPA technique in which copy number detection is combined with the use of a methylation-sensitive restriction enzyme [26]. Probe sequences, gene loci, and chromosome locations can be found at http://www.mlpa.com. The experimental procedure was carried out according to the manufacturer's instructions. Reaction products were separated on an automated sequencer (ABI 310 capillary) and visualised with Genemapper analysis v.4.0 (Applied Biosystems). Values corresponding to peak size (base pairs) and peak height were used for further data processing by Coffalyser V7 software (MRC-Holland). All MS-MLPA reactions were performed at least two times. The methylation profile of each sample was assessed according to MRC-Holland instructions.

Aberrant methylation was scored as a categorical variable using a specific Methylation Ratio (MR) for each gene corresponding to the highest level of accuracy of the test, according to previously reported [27].
2.8. LINE-1 PCR and Pyrosequencing. The methylation status of LINE-1 was evaluated by bisulfite-PCR and pyrosequencing [28] in all tumors and in twenty-five samples of normal colonic mucosa. Thirteen of these samples were derived from normal tissue at the resection margins of 13 patients with sporadic CRCs. The remaining normal specimens were obtained from 12 individuals who had undergone surgery for ischemic colorectal disease or for diverticulitis without a personal history of colorectal cancer. Bisulfite treatment of genomic DNA converts all unmethylated cytosines into thymine while methylated cytosines remain unchanged. All the cytosine residues unconverted in the sequence represent methylated cytosines in the genome. In this method, $1.5 \mathrm{mg}$ of DNA was denatured in $50 \mathrm{~mL}$ of $0.2 \mathrm{M} \mathrm{NaOH}$ for $10 \mathrm{~min}$ at $37^{\circ} \mathrm{C}$. Then, $30 \mathrm{~mL}$ of freshly prepared $10 \mathrm{mM}$ hydroquinone and $520 \mathrm{~mL}$ of $3 \mathrm{M}$ sodium bisulfite at $\mathrm{pH} 5.0$ were added and mixed. The samples were incubated at $50^{\circ} \mathrm{C}$ for $16 \mathrm{~h}$. The bisulfite-treated DNA was purified using Wizard DNA CleanUp System (Promega).

LINE-1 assay was designed toward a consensus LINE-1 sequence (GenBank accession number X58075) and allowed to quantify the percentage of 5-methylated cytosines $(\% 5 \mathrm{mC})$ in five consecutive $\mathrm{CpG}$ sites. PCR was performed in a $50 \mu \mathrm{L}$ reaction volume that included 2 pmol of forward primer $5^{\prime}$-GAGTTAGGTGTGGGATATAGT-3', 2 pmol of reverse biotinylated primer $5^{\prime}$-CAAAAAATCAAAAAATTCCCTTCCC-3', $5 \mu \mathrm{L}$ of bisulfite-treated genomic DNA 1.25 units of GoTaq DNA polymerase, 1X GoTaq Flexi Buffer (Promega, Madison, WI, USA), and $200 \mu \mathrm{M}$ dNTPs. Thermal cycling conditions were $3 \mathrm{~min}$ at $95^{\circ} \mathrm{C}, 45$ cycles at $95^{\circ} \mathrm{C} / 25 \mathrm{~s}, 50^{\circ} \mathrm{C} / 25 \mathrm{~s}$, and $72^{\circ} \mathrm{C} / 25 \mathrm{~s}$, followed by a final extension at $72^{\circ} \mathrm{C}$ for $5 \mathrm{~min}$. Pyrosequencing was performed on PCR product with bound LINE-1 sequencing primer $5^{\prime}$-GGTGTGGGATATAGTT-3', according to the protocol reported above. Fully methylated DNA (CpGenome Universal Methylated DNA, Millipore, Billerica, MA, USA) and unmethylated DNA (CpGenome Universal Unmethylated DNA, Millipore, Billerica, MA, USA) were used as positive and negative controls for optimizing the assay.

2.9. Statistical Analysis. Univariate comparisons of continuous data were carried out using Student's $t$-test and discrete variables were compared with $\chi^{2}$ test or Fisher's exact test. The association between discrete outcome and continuous predictor was evaluated with a logistic regression model. All comparisons were two-sided and a $p$ value $<0.05$ was considered to be significant.

\section{Results}

3.1. Patients Features. We recruited a total of 38 patients with adenocarcinoma in the colon-rectum diagnosed before the age of 40 years, but the biological material was available for 33 patients. Clinicopathological features of the 33 colorectal adenocarcinomas and patients are shown in Table 1 . The mean age of disease onset was 35 years. We observed that the male gender was approximately 3 times more frequent than female (76\% versus $24 \%$ ), tumor location was preferentially the left 
TABLE 1: Clinicopathological features of 33 colorectal adenocarcinomas (and patients) developed $\leq 40$ years (1984-2008) compared to patients with cancer onset $>60$ years.

\begin{tabular}{|c|c|c|c|}
\hline & Technical details & $\begin{array}{l}\text { Patients with onset } \leq 40 \text { years, } \\
\qquad N=33(\%)\end{array}$ & $\begin{array}{l}\text { Patients with onset }>60 \text { years, } \\
\qquad N=41(\%)\end{array}$ \\
\hline \multirow{3}{*}{ Age of onset of disease } & Min & 11 & 61 \\
\hline & $\operatorname{Max}$ & 40 & 85 \\
\hline & Mean & 35 & 73 \\
\hline \multirow{2}{*}{ Sex } & Female & $8(24)$ & $11(27)$ \\
\hline & Male & $25(76)$ & $30(73)$ \\
\hline \multirow{3}{*}{$\begin{array}{l}\text { Tumor location in the large } \\
\text { bowel }\end{array}$} & Right colon & $7(21)$ & $12(29)$ \\
\hline & Left colon & $17(52)$ & $19(47)$ \\
\hline & Rectum & $9(27)$ & $10(24)$ \\
\hline \multirow{4}{*}{ Stage (Dukes) } & $\mathrm{A}$ & $5(15)$ & $7(17)$ \\
\hline & $\mathrm{B}$ & $7(21)$ & $9(22)$ \\
\hline & $\mathrm{C}$ & $12(37)$ & $14(34)$ \\
\hline & $\mathrm{D}$ & $9(27)$ & $11(27)$ \\
\hline \multirow{3}{*}{ Tumor differentiation } & Low-grade & $19(58)$ & $31(76)$ \\
\hline & High-grade & $8(24)$ & $8(19)$ \\
\hline & Mucinous & $6(18)$ & $2(5)$ \\
\hline
\end{tabular}

colon (52\%), and C and D stages of Dukes (37\% and $27 \%$, resp.) were more frequently represented. Fifty-eight percent of early onset tumors were well or moderately differentiated. Family history of colorectal cancer was present in $8(24 \%)$ patients: 2 with Bethesda criteria (patients with a first-degree relative affected by colorectal cancer), 5 who fulfilled the Amsterdam II criteria, and 1 with Familial Adenomatous Polyposis. Clinical features of Cowden/Bannayan [29] and Peutz-Jeghers syndromes [30] were not identified in any patients.

3.2. MMR Deficiency and Somatic Mutations of KRAS and BRAF Genes. MMR deficiency was evaluated by both immunohistochemistry and MSI analysis in all 74 colorectal cancers. MMR deficiency was defined as loss of protein expression in any of the MMR proteins and/or having a MSI tumor. Seven out of 33 (21\%) early onset tumors showed MSI and loss of expression of MMR proteins ( 4 for MLH1/PMS2 and 3 for MSH2/MSH6). By family history, 5 patients $(\leq 40$ years) with MMR deficient tumors fulfilled clinical features of Lynch syndrome and 2 the Bethesda Criteria. Four out of $41(10 \%)$ tumors in the control group (4 for MLH1/PMS2) were MMR deficient. These 4 tumors diagnosed over the age of 60 years showed somatic $M L H 1$ hypermethylation and V600E mutation in BRAF gene. No V600E mutations were detected in MSS tumors with clinical onset at advanced age or in early onset colorectal cancers. We found somatic MSH2 methylation in only one case (patient with germinal EPCAM deletion) among 7 young patients showing MSI and loss of MSH2/MSH6 protein expression. KRAS mutations were found in 10 early onset tumors and in 4 cases of the control group. Thus, tumors of juvenile cases showed more often somatic KRAS mutations (30\% versus $10 \%$ ). Molecular results in the investigated patients are summarized in Table 2.
3.3. Germline MMR, APC, and MUTYH Mutations. We identified 6 cases with sequence variants in either $M L H 1$ (3 cases), MSH2 (1 case), EPCAM (1 case), or APC (1 case) (Table 3). For $M L H 1$, we detected one insertion mutation (p.Arg497ProfsX6) and two missense mutations (p.Leu749Pro and p.Glu663Asp). These two missense variants were classified as Class 5 mutations (>99\% likelihood of pathogenicity) by Plon et al. [31]. For $M S H 2$, we found one nonsense mutation (p.Phe294X). Moreover, we identified a large deletion of exons 8 and 9 in EPCAM gene, without involvement of $\mathrm{MSH} 2$ promoter, using the P003-B2 MLPA kit. This finding was confirmed by the P072-B1 MLPA kit (MRC-Holland). Only one patient was affected by Familial Adenomatous Polyposis and showed an insertion mutation in $A P C$ gene at exon 15 (p.Arg924SerfsX16). Germline analysis of MUTYH gene was carried out in the whole group of young patients, but no monoallelic or biallelic alteration was detected.

3.4. Gene-Specific DNA Methylation. MS-MLPA assay was employed to evaluate the hypermethylation profiles relative to tumors from early onset patients $(n=33)$ and control group $(n=41)$. The average number of methylated genes was significantly higher in the control group compared with the young group (5.025 versus 3.3, resp.) ( $p=0.02$, Figure 1(a)). By contrast, no significant differences were observed between young and hereditary/suspect of hereditary cases (Figure 1(b)).

In the control group, we observed two main tumor sets on the basis of the degree of methylation. The first group consisted of 7 CRCs (17\% of cases) showing high levels of gene methylation, involving a mean percentage of $28 \%$ of the promoters examined (ranging from $24 \%$ to $37 \%$ ). The second group included the remaining $34 \mathrm{CRCs}$ showing absent or low level methylation involving a mean percentage of $9 \%$ of the genes analysed (ranging from $0 \%$ to 20\%) (Figure 2). 
TABLE 2: Molecular features of colorectal carcinomas in cases and in the control group.

\begin{tabular}{|c|c|c|c|}
\hline \multirow{3}{*}{ Technical detail } & \multicolumn{2}{|c|}{ Patients with onset $\leq 40$ years } & \multirow{3}{*}{ Control group $>60$ years, $N=41(\%)$} \\
\hline & \multicolumn{2}{|c|}{$N=33$} & \\
\hline & $\begin{array}{c}\text { Nonhereditary cases, } \\
N=25(\%)\end{array}$ & $\begin{array}{c}\text { Hereditary/suspect of hereditary } \\
\text { cases, } N=8(\%)\end{array}$ & \\
\hline \multicolumn{4}{|l|}{ MMR alterations } \\
\hline $\begin{array}{l}\text { MSI and no expression } \\
\text { MMR proteins }\end{array}$ & 0 & $7(88)$ & $4(10)$ \\
\hline \multicolumn{4}{|l|}{ Methylation } \\
\hline $\begin{array}{l}M L H 1 \text { or } M S H 2 \text { promoter } \\
\text { hypermethylation }\end{array}$ & 0 & $1(13)$ & $4(10)$ \\
\hline \multicolumn{4}{|l|}{ Somatic mutations } \\
\hline KRAS mutation & $9(36)$ & $1(13)$ & $4(10)$ \\
\hline$B R A F$ mutation & 0 & 0 & $4(10)$ \\
\hline \multicolumn{4}{|l|}{ Germline mutations } \\
\hline$M M R$ & 0 & $5(63)$ & 0 \\
\hline$A P C$ & 0 & $1(13)$ & 0 \\
\hline MUTYH & 0 & 0 & 0 \\
\hline
\end{tabular}

TABLE 3: Constitutional mutations in early onset colorectal cancer.

\begin{tabular}{|c|c|c|c|}
\hline & Cases & Gene & Mutation \\
\hline \multirow{5}{*}{ Lynch syndrome } & \multirow{5}{*}{5} & & c.2246T>C; p.Leu749Pro \\
\hline & & MLH1 (3) & c.1489dupC; p.Arg497ProfsX6 \\
\hline & & & c.1989G > T; p.Glu663Asp \\
\hline & & MSH2 (1) & c.881_882delTT; p.Phe294X \\
\hline & & EPCAM (1) & Del ex $8-9$ and seq in $3^{\prime}$ to $+3 \mathrm{~kb}$ \\
\hline Familial Adenomatous Polyposis & 1 & $A P C$ & c.2771_2772insT; p. Arg924SerfsX16 \\
\hline
\end{tabular}

The seven CRCs exhibiting extensive gene methylation included all the cases showing MSI and BRAF mutation and MLH1 methylation.

On the other hand, in the group of early onset tumors, we did not detect any case with extensive gene methylation. No methylation for the $M M R$ genes was found in these tumors, with the exception of the case with EPCAM gene mutation (A34), which was methylated in the $\mathrm{MSH} 2$ gene. Evaluating the hypermethylation frequency in single genes, no significant differences were observed comparing early onset patients and control group. By contrast, a very high frequency of methylation was detected in ESR1, GATA5, and WT1 genes in both groups of the familiarity (Figure 3 ).

3.5. LINE-1 Hypomethylation. We used the quantitative bisulfite pyrosequencing to determine the methylation status of LINE-1 repetitive sequences in all CRCs compared to twentyfive samples of normal colonic mucosa. In normal samples, LINE-1 methylation levels were high (average 60.78\% $\pm 0.4 \%$ ) and very similar to those commonly observed in peripheral blood cells from normal individuals [32]. By contrast, LINE1 methylation levels in CRCs were significantly lower than in normal samples (mean 55.4\% $\pm 0.86 ; p<0.024$ ).

Mean LINE-1 methylation levels in the three study groups of colorectal cancers were early onset, 54.3\%; late onset,
55.9\%; Lynch syndrome, 59.0\% (Figure 4). The difference of LINE-1 hypomethylation in early onset colorectal cancer was not significant when compared to late onset ones. Interestingly, in the Lynch syndrome tumors LINE-1 methylation levels were higher than early onset and late onset groups and similar in the mean percentage of normal mucosa. However, this difference did not reach statistical significance because of the small number of Lynch tumors.

\section{Discussion}

In this study we have assessed the clinicopathological, molecular, and familial features of 33 early onset colorectal cancers ( $\leq 40$ years). We showed that the frequency of known hereditary colorectal cancer syndromes in this population was $18 \%$. This cohort disclosed a molecular profile of $M M R$ deficiency characterised by germline mutations in $M L H 1, M S H 2$, and EPCAM genes, thus confirming that Lynch syndrome is the most frequent cause of hereditary colorectal cancer in young patients $[12,19]$.

Previous studies have revealed that colorectal cancer diagnosed at early ages had a high probability of showing MMR deficiency, ranging from $26 \%$ to $73 \%$ [11]. The reason for this wide range could be explained by the different age thresholds (from 24 to 50 years) and by diverse panels of 


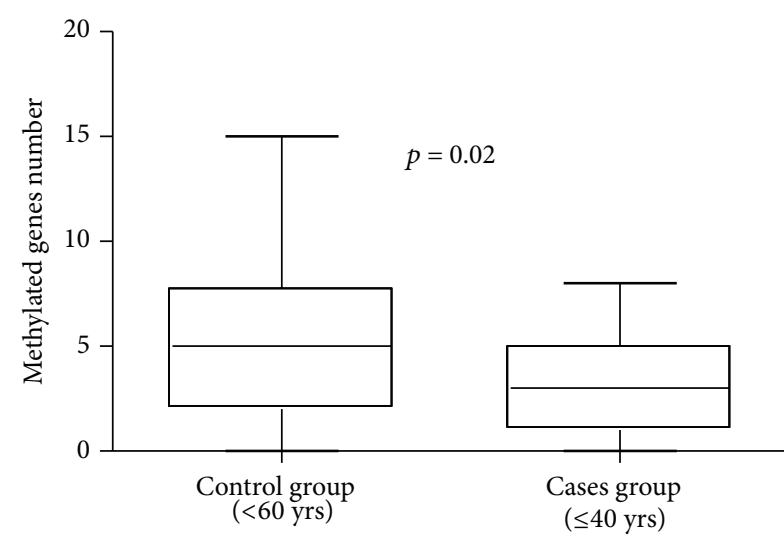

(a)

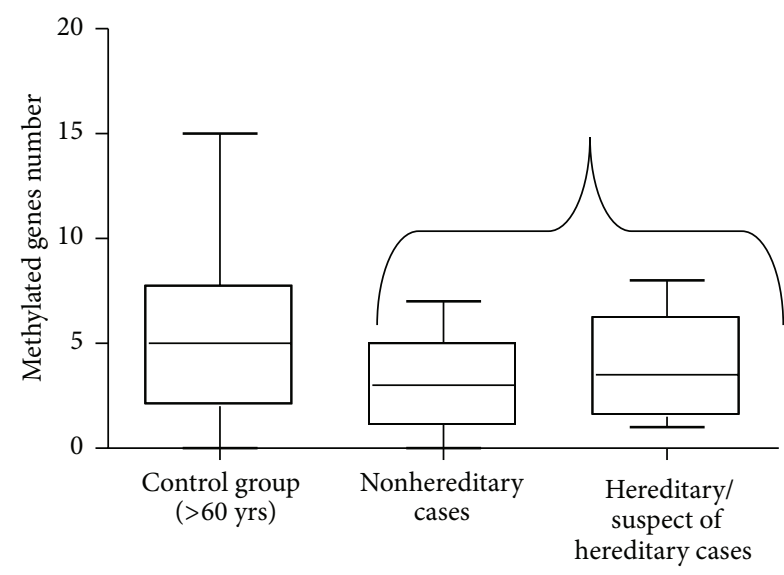

(b)

FIGURE 1: Results of methylation status in young group and in elderly group. In (a), we observed a significant difference in methylation pattern between patients under 40 yrs and patients over 60 yrs. The mean number of methylated genes in the control group is higher than average number in the cases group. In (b), we observe no statistical difference between control, young, and hereditary cases, but the difference remained significant between patients under $40 \mathrm{yrs}$ and patients over $60 \mathrm{yrs}$.

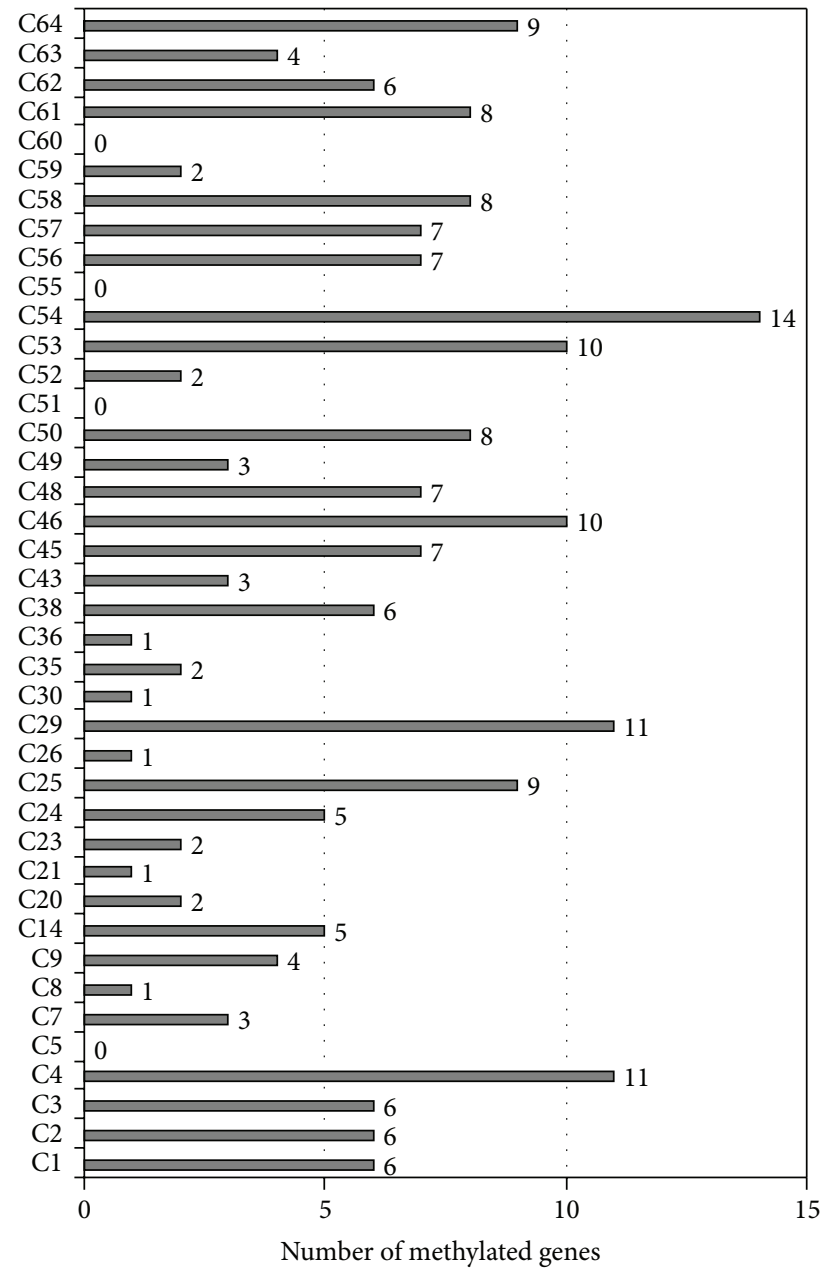

Control cases

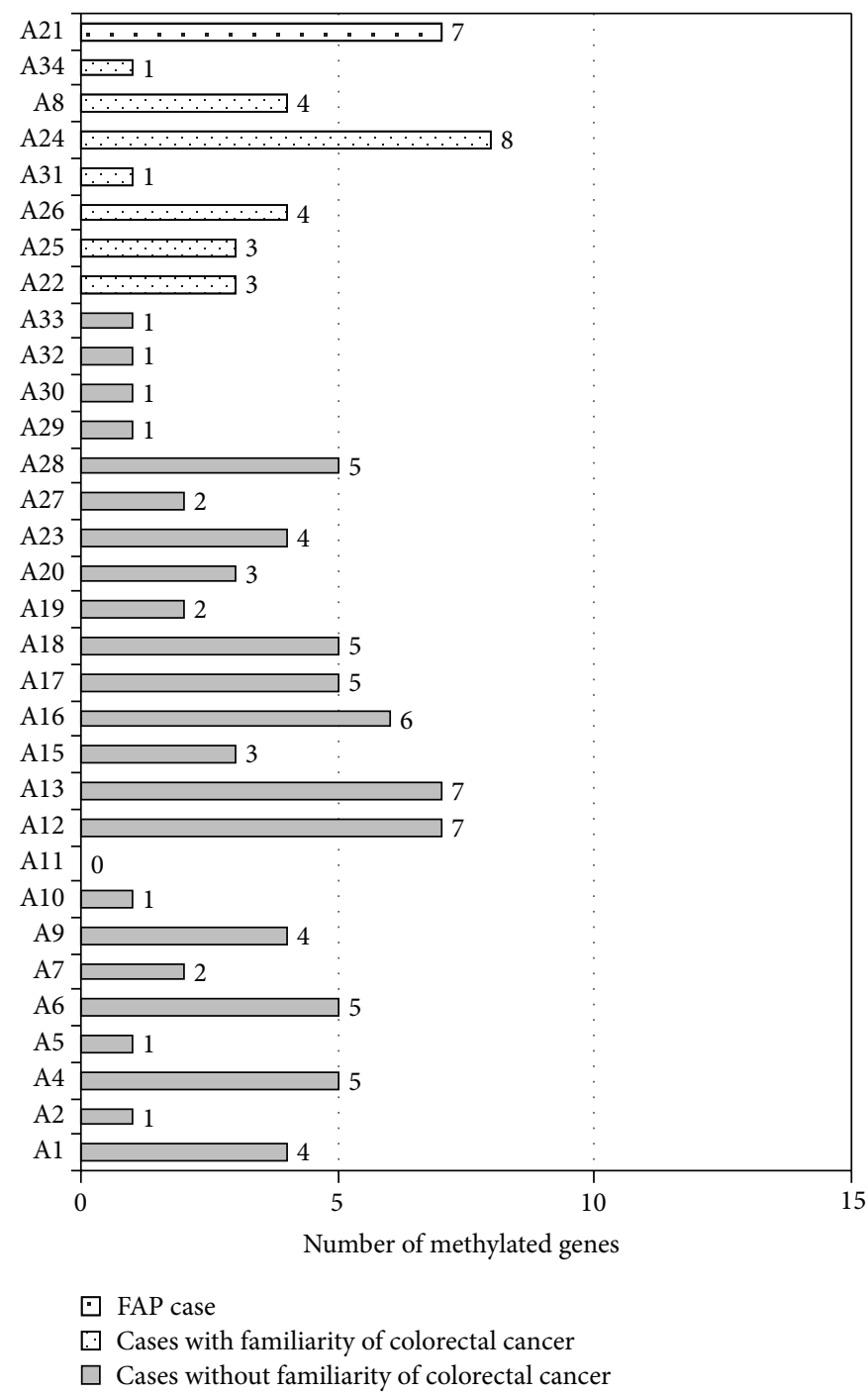

FIGURE 2: Number of methylated genes in control cases and in early onset colorectal cancer. 


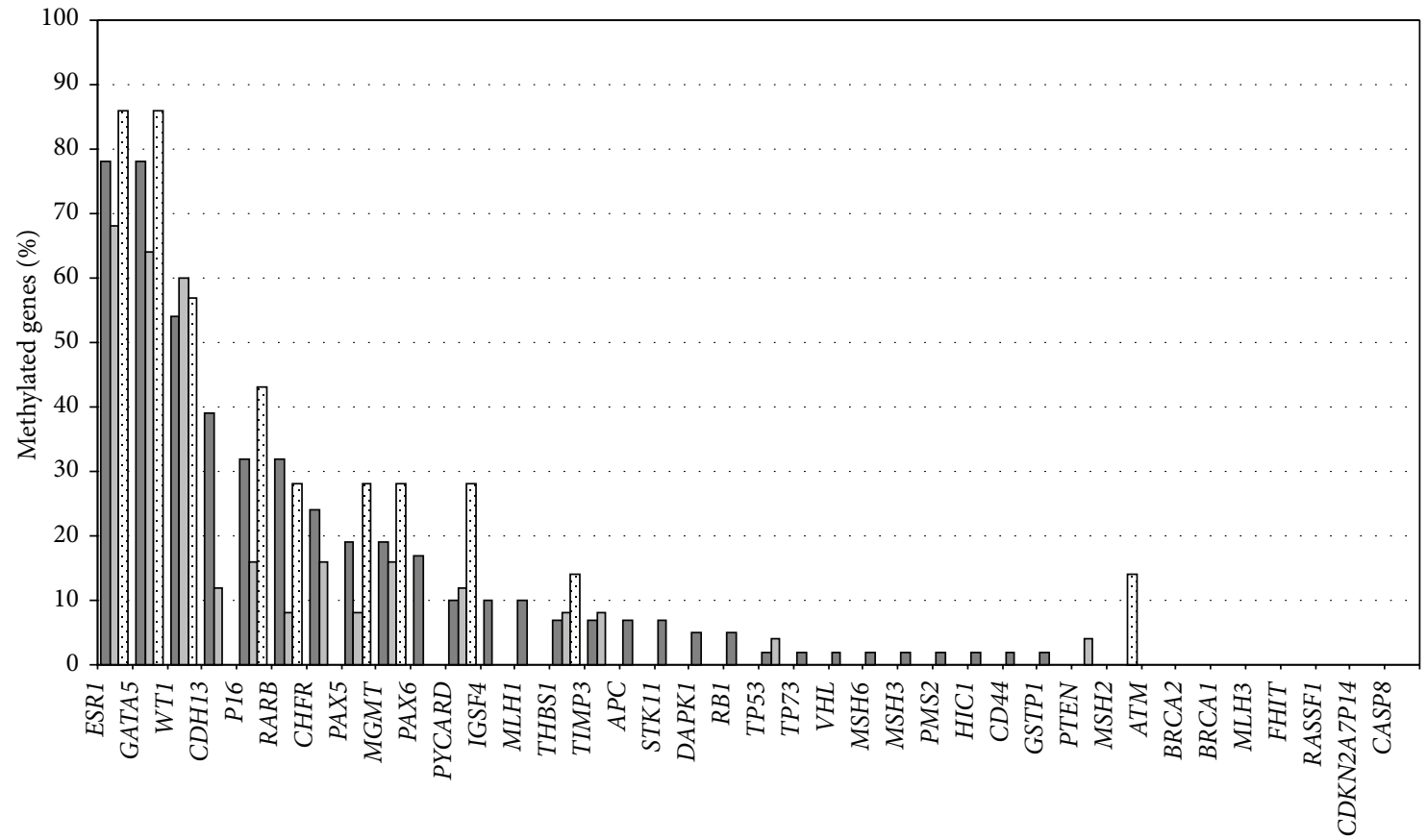

Methylated gene

$\square$ Control cases

$\square$ Cases without familiarity of colorectal cancer

๑. Cases with familiarity of colorectal cancer

FIGURE 3: Hypermethylation frequency in single genes.

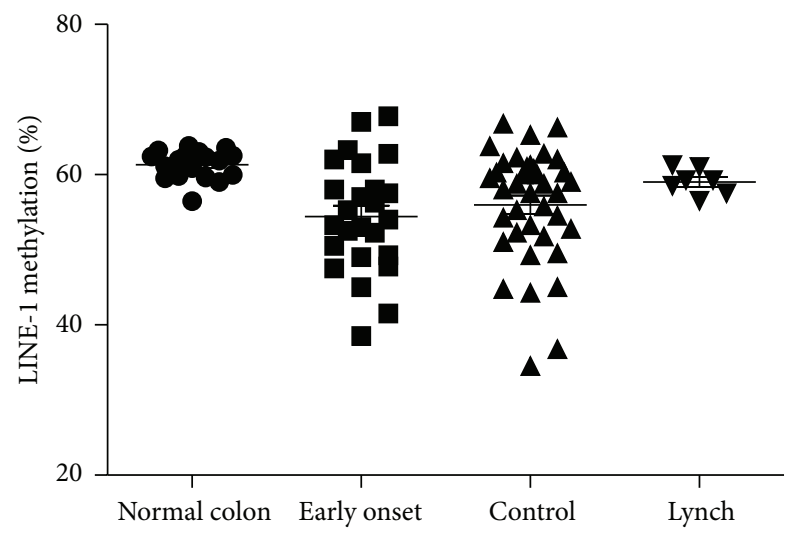

FIGURE 4: LINE-1 hypomethylation in early and late onset patients.

proteins analysed by IHC (usually restricted to MLH1 and MSH2). Moreover, the majority of studies have analysed either Lynch syndrome or sporadic colorectal cancer and it is known that the rate of MSI is much lower in the latter. Our study, based on a population based approach of colorectal cancer developed $\leq 40$ years recruited from the specialised Colorectal Cancer Registry of Modena, showed that the frequency of MMR deficiency was $21 \%$. Although we identified 7 tumors with MMR deficiency, we detected only five patients with pathogenetic germline mutations in $M M R$ genes. Possible causes for the lack of identified constitutional mutations could be low sensitivity of analytical methods or genetic events [33] that affect both alleles of a $M M R$ gene. We did not identify any carrier of MSH6 germline mutations. Recent studies have shown that the average age of colorectal cancer onset in MSH6 mutation carriers has been estimated to be around 50 years, while $M L H 1$ and $M S H 2$ carriers are diagnosed on average 10 years earlier [34]. Moreover, mutations in the MSH6 gene have also been linked to a lower risk of colorectal cancer and a higher risk of endometrial carcinoma [34]. Although germline mutations of MSH6 gene in early onset colorectal cancer have been reported [35], this difference in age of onset and associated risk may explain why MSH6 mutations constitute a minor fraction of cases.

In summary, as suggested by Jasperson et al. [11], the study of family cancer history, MSI, and IHC analyses followed by germline genetic testing represent an effective procedure for the diagnosis of Lynch syndrome in early onset cases.

Some population based studies showed that $~ 30 \%$ of biallelic MUTYH mutation carriers develop a colorectal cancer in the absence of a polyposis phenotype $[13,16,35]$. Accordingly, it has been suggested that MUTYH testing should be considered in early onset colorectal cancer patients with intact DNA MMR, regardless of family history or number of colonic polyps [13]. Giráldez et al. detected 2.8\% of biallelic MUTYH mutations in a cohort of 140 patients with colorectal cancer diagnosed before the age of 50 [36]. In our study we performed systematic whole-gene sequencing 
and did not find biallelic or monoallelic MUTYH mutations. These negative results could be explained by the limited number of investigated cases.

We identified only one pathogenetic mutation of $A P C$ in a 34-year-old patient with colorectal cancer and polyposis. The literature describes that Familial Adenomatous Polyposis is responsible for less than $1 \%$ of all colorectal cancers [37] and that the mean age for colorectal cancer development in this group of individuals is approximately 39 years [38], suggesting that also $A P C$ gene is implicated in cancer occurring at an early age.

In agreement with that reported in literature, we observed that $82 \%$ of early onset CRCs were not associated with known hereditary CRC syndromes [36]. This subset of tumors was mainly characterized by distal location, advanced stage, and predominance of the male gender as well as other investigators supported [39]. A previous study according to Ahnen et al. reported that cancer-specific survival in patients with young onset CRC is comparable to that of patients with late onset cancer $[5,38]$. Moreover, we observed a similar frequency of somatic mutations for KRAS oncogene to overall sporadic colorectal cancers that correspond to 40\% [40]. Regarding $B R A F$ gene, a recent study suggests that $B R A F$ mutations occur in $10-20 \%$ of sporadic colorectal cancer and are closely associated with the MLH1 methylation. In our young patients, no BRAF mutations or MLH1 promoter methylation was detected.

A second important aspect of our work concerns the analysis of aberrant hypo- and hyper-DNA methylation profiles of early onset CRCs compared with late onset CRCs. Both of these alterations are widely accepted as potential source of early biomarkers for diagnosis and prognosis in CRCs. CIMP phenotype, which accounts for almost 30-40\% [41], has rarely been evaluated in early onset CRCs. In our work, no case with extensive gene methylation was observed among early CRCs and the average number of methylated genes was significantly lower in these tumors (both hereditary and nonhereditary CRCs) compared with the control group.

These results are in agreement with previous works that reported low levels of gene hypermethylation in Lynch syndrome cases $[42,43]$ as well as in nonhereditary early onset CRCs [19].

In our study, extensive gene hypermethylation was observed only in the late onset CRCs, accounting for $17 \%$ of these cases. In agreement with literature, this subset of CRCs frequently showed MSI and BRAF mutation and MLH1 methylation $[41,43]$.

For the first time, we demonstrated that hypermethylation of three genes, namely, ESR1, GATA5, and WT1, was very common both in early onset (hereditary and nonhereditary tumors) and in late onset CRCs. Although these results need to be validated with further studies, our data have important clinical implications suggesting the usefulness of aberrant gene methylation analysis for the early detection and risk assessment of CRC, without using age at onset as a differential criterion. Promoter methylation analysis of serum and stool DNA has the potential to be used as a noninvasive test for the early diagnosis of CRC [44]. However, accurate selection of methylation markers is crucial for sensitive and specific detection of CRC as de novo methylation is also associated with aging [45]. ESR1 is a well-known "type A" (age related) gene because its hypermethylation is demonstrated in both normal colorectal mucosa and CRCs, proportional to tissue age. By contrast, GATA5 methylation has been reported as a suitable marker for early diagnosis of CRC [46] and its methylation is observed in colorectal adenomas but not in inflammatory colorectal tissues.

Moreover, our analysis has highlighted the potential utility of the WT1 gene as an early diagnostic marker of CRC. To date, only a few studies have investigated WT1 methylation, confirming our data of widespread methylation of this gene in CRCs $[47,48]$. In our opinion, this finding deserves to be explored further, especially with respect to the aberrant mechanisms of loss of imprinting of $11 p 15$ described in CRCs and the possible link between WT1 methylation and the upregulation of IGF2 transcription [49].

Genome-wide hypomethylation is also reported as an early event in CRC and it has been associated with the activation of protooncogenes (i.e., MET) [50] and the presence of chromosomal instability [51]. Recently, Antelo et al. [39] found significantly lower levels of LINE-1 methylation in early onset CRCs compared to late onset CRCs, suggesting that a high degree of LINE-1 hypomethylation is a unique feature of CRCs in young patients. At variance, our study does not confirm these previous findings, demonstrating very similar LINE-1 methylation levels in early onset and in late onset CRCs, with a normal distribution of LINE-1 values in both subsets of tumors. In our opinion, several factors may explain this discordance. Firstly, since the degree of LINE-1 demethylation prognosis is linear in relation to TNMstage progression and this marker is a strong independent factor for poor prognosis [52], the evaluation of tumor stage is crucial when comparing different subsets of CRCs. The second important point regards the tumor location because lower levels of LINE-1 methylation have been reported in distal compared with proximal CRCs [39]. Antelo et al. examined two independent cohorts of CRCs developed $\leq 50$ years including mainly advanced and distal CRCs without a matched selection of the late onset CRCs. For these reasons we designed a case-control study in which every early onset CRC was matched with a late onset CRC for sex, location, and stage. Finally, technical reasons may not be excluded, although the same methodological approach has been used in the two studies and very similar LINE-1 methylation levels were observed considering normal colorectal mucosa and MSI CRCs (both hereditary and sporadic tumors).

Moreover, Antelo et al. reported a similar result about higher levels of LINE-1 methylation in Lynch syndrome tumors than in group of early onset CRC. For this reason, it is maybe not optimal to use this testing for detection of early onset CRC, whereas Lynch syndrome is the most common hereditary CRC in young patients. Although our series of early onset CRCs is small and the present results are not conclusive about this issue, we believe that the strong positive association between LINE-1 hypomethylation and early onset CRC previously reported needs to be reconsidered through future larger case-control studies. 


\section{Conclusion}

The results of this study can be summarised as follows.

First, Lynch syndrome is the most frequent cause of hereditary colorectal cancer in young patients; family cancer history, MSI, and IHC analyses followed by germline genetic testing represent the most appropriate procedure for Lynch syndrome diagnosis in early onset colorectal cancer. Second, early onset colorectal cancers with MMR deficiency were clinically and pathologically indistinguishable from colorectal MSS carcinomas. Third, epigenetic events (hyperand hypomethylation) are not closely associated with early onset colorectal cancer. Finally, our study emphasises that the genetic basis in the majority of early onset colorectal carcinomas remains unknown. Further studies of the whole exome of this genetically undefined group of early onset colorectal tumors will need to elucidate possible pathogenetic mechanisms.

\section{Conflict of Interests}

The authors declare that there is no conflict of interests regarding the publication of this paper.

\section{Acknowledgments}

This work has been carried out with the financial support of Associazione Italiana Ricerca Cancro (AIRC) and the Associazione Ricerca Tumori Intestinali (ARTI). The authors gratefully acknowledge Maurizio Ponz de Leon for his expertise in hereditary colorectal cancer and Carmela Di Gregorio for her help in collecting samples and immunohistochemical analysis.

\section{References}

[1] H. Raskov, H. C. Pommergaard, J. Burcharth, and J. Rosenberg, "Colorectal carcinogenesis-update and perspectives," World Journal of Gastroenterology, vol. 20, no. 48, pp. 18151-18164, 2014.

[2] J. Weitz, M. Koch, J. Debus, T. Höhler, P. R. Galle, and M. W. Büchler, "Colorectal cancer," The Lancet, vol. 365, no. 9454, pp. 153-165, 2005.

[3] J. E. Meyer, T. Narang, F. H. Schnoll-Sussman, M. B. Pochapin, P. J. Christos, and D. L. Sherr, "Increasing incidence of rectal cancer in patients aged younger than 40 years: an analysis of the surveillance, epidemiology, and end results database," Cancer, vol. 116, no. 18, pp. 4354-4359, 2010.

[4] R. Siegel, D. Naishadham, and A. Jemal, "Cancer statistics, 2012," CA: A Cancer Journal for Clinicians, vol. 62, no. 1, pp. 10-29, 2012.

[5] F. Domati, S. Maffei, S. Kaleci et al., "Incidence, clinical features and possible etiology of early onset ( $\leq 40$ years) colorectal neoplasms," Internal and Emergency Medicine, vol. 9, no. 6, pp. 623-631, 2013.

[6] S. Ganapathi, D. Kumar, N. Katsoulas et al., "Colorectal cancer in the young: trends, characteristics and outcome," International Journal of Colorectal Disease, vol. 26, no. 7, pp. 927-934, 2011.

[7] L. Losi, C. Di Gregorio, M. Pedroni et al., "Molecular genetic alterations and clinical features in early-onset colorectal carcinomas and their role for the recognition of hereditary cancer syndromes," American Journal of Gastroenterology, vol. 100, no. 10, pp. 2280-2287, 2005.

[8] J. E. Meyer, T. Narang, F. H. Schnoll-Sussman, M. B. Pochapin, P. J. Christos, and D. L. Sherr, "Increasing incidence of rectal cancer in patients aged younger than 40 years: an analysis of the surveillance, epidemiology, and end results database," Cancer, vol. 116, no. 18, pp. 4354-4359, 2010.

[9] D. T. Chang, R. K. Pai, L. A. Rybicki et al., "Clinicopathologic and molecular features of sporadic early-onset colorectal adenocarcinoma: an adenocarcinoma with frequent signet ring cell differentiation, rectal and sigmoid involvement, and adverse morphologic features," Modern Pathology, vol. 25, no. 8, pp. 1128-1139, 2012.

[10] R. Gryfe, H. Kim, E. T. K. Hsieh et al., “Tumor microsatellite instability and clinical outcome in young patients with colorectal cancer," The New England Journal of Medicine, vol. 342, no. 2, pp. 69-77, 2000.

[11] K. W. Jasperson, T. M. Vu, A. L. Schwab et al., "Evaluating Lynch syndrome in very early onset colorectal cancer probands without apparent polyposis," Familial Cancer, vol. 9, no. 2, pp. 99-107, 2010.

[12] L. Sehofield, N. Watson, F. Grieu et al., "Population-based detection of lynch syndrome in young colorectal cancer patients using microsatellite instability as the initial test," International Journal of Cancer, vol. 124, no. 5, pp. 1097-1102, 2009.

[13] F. Balaguer, S. Castellví-Bel, A. Castells et al., "Identification of MYH mutation carriers in colorectal cancer: a multicenter, case-control, population-based study," Clinical Gastroenterology and Hepatology, vol. 5, no. 3, pp. 379-387, 2007.

[14] T. J. McGarrity, H. E. Kulin, and R. J. Zaino, "Peutz-Jeghers syndrome," The American Journal of Gastroenterology, vol. 95, no. 3, pp. 596-604, 2000.

[15] R. Pilarski, J. A. Stephens, R. Noss, J. L. Fisher, and T. W. Prior, "Predicting PTEN mutations: an evaluation of cowden syndrome and Bannayan-Riley-Ruvalcaba syndrome clinical features," Journal of Medical Genetics, vol. 48, no. 8, pp. 505-512, 2011.

[16] S. M. Farrington, A. Tenesa, R. Barnetson et al., "Germline susceptibility to colorectal cancer due to base-excision repair gene defects," American Journal of Human Genetics, vol. 77, no. 1, pp. 112-119, 2005.

[17] M. Berg, S. A. Danielsen, A. Terje et al., "DNA sequence profiles of the colorectal cancer critical gene set KRAS-BRAF-PIK3CAPTEN-TP53 related to age at disease onset," PLOS ONE, vol. 5, no. 11, Article ID e13978, 2010.

[18] J. P. Terdiman, T. R. Levin, B. A. Allen et al., "Hereditary nonpolyposis colorectal cancer in young colorectal cancer patients: high-risk clinic versus population-based registry," Gastroenterology, vol. 122, no. 4, pp. 940-947, 2002.

[19] J. Perea, D. Rueda, A. Canal et al., "Age at onset should be a major criterion for subclassification of colorectal cancer," The Journal of Molecular Diagnostics, vol. 16, no. 1, pp. 116-126, 2014.

[20] S. B. Edge and C. C. Compton, "The American Joint Committee on Cancer: the 7th edition of the AJCC cancer staging manual and the future of TNM," Annals of Surgical Oncology, vol. 17, no. 6, pp. 1471-1474, 2010.

[21] M. Pedroni, B. Roncari, S. Maffei et al., "A mononucleotide markers panel to identify hMLH1/hMSH2 germline mutations," Disease Markers, vol. 23, no. 3, pp. 179-187, 2007.

[22] N. Suraweera, A. Duval, M. Reperant et al., "Evaluation of tumor microsatellite instability using five quasimonomorphic 
mononucleotide repeats and pentaplex PCR," Gastroenterology, vol. 123, no. 6, pp. 1804-1811, 2002.

[23] R. M. Xicola, X. Llor, E. Pons et al., "Performance of different microsatellite marker panels for detection of mismatch repairdeficient colorectal tumors," Journal of the National Cancer Institute, vol. 99, no. 3, pp. 244-252, 2007.

[24] V. Deschoolmeester, M. Baay, W. Wuyts et al., "Detection of microsatellite instability in colorectal cancer using an alternative multiplex assay of quasi-monomorphic mononucleotide markers," Journal of Molecular Diagnostics, vol. 10, no. 2, pp. 154-159, 2008.

[25] M. P. Quinlan and J. Settleman, "Isoform-specific ras functions in development and cancer," Future Oncology, vol. 5, no. 1, pp. 105-116, 2009.

[26] A. O. H. Nygren, N. Ameziane, H. M. B. Duarte et al., "Methylation-specific MLPA (MS-MLPA): simultaneous detection of CpG methylation and copy number changes of up to 40 sequences," Nucleic Acids Research, vol. 33, no. 14, article e128, 2005.

[27] D. Furlan, N. Sahnane, B. Bernasconi et al., "APC alterations are frequently involved in the pathogenesis of acinar cell carcinoma of the pancreas, mainly through gene loss and promoter hypermethylation," Virchows Archiv, vol. 464, pp. 553-564, 2014.

[28] A. S. Yang, M. R. H. Estécio, K. Doshi, Y. Kondo, E. H. Tajara, and J. P. J. Issa, "A simple method for estimating global DNA methylation using bisulfite PCR of repetitive DNA elements," Nucleic Acids Research, vol. 32, no. 3, article e38, 2004.

[29] M.-H. Tan, J. Mester, C. Peterson et al., "A clinical scoring system for selection of patients for pten mutation testing is proposed on the basis of a prospective study of 3042 probands," American Journal of Human Genetics, vol. 88, no. 1, pp. 42-56, 2011.

[30] A. D. Beggs, A. R. Latchford, H. F. A. Vasen et al., "PeutzJeghers syndrome: a systematic review and recommendations for management," Gut, vol. 59, no. 7, pp. 975-986, 2010.

[31] S. E. Plon, D. M. Eccles, D. Easton et al., "Sequence variant classification and reporting: recommendations for improving the interpretation of cancer susceptibility genetic test results," Human Mutation, vol. 29, no. 11, pp. 1282-1291, 2008.

[32] Y. Baba, C. Huttenhower, K. Nosho et al., "Epigenomic diversity of colorectal cancer indicated by LINE-1 methylation in a database of 869 tumors," Molecular Cancer, vol. 9, article 125, 2010.

[33] A. R. Mensenkamp, I. P. Vogelaar, W. A. G. van Zelst-Stams et al., "Somatic mutations in MLH1 and MSH2 are a frequent cause of mismatch-repair deficiency in lynch syndrome-like tumors," Gastroenterology, vol. 146, no. 3, pp. 643-646, 2014.

[34] J. Plaschke, C. Engel, S. Krüger et al., "Lower incidence of colorectal cancer and later age of disease onset in 27 families with pathogenic MSH6 germline mutations compared with families with MLH1 or MSH2 mutations: the German hereditary nonpolyposis colorectal cancer consortium," Journal of Clinical Oncology, vol. 22, no. 22, pp. 4486-4494, 2004.

[35] C. Pinto, I. Veiga, M. Pinheiro et al., "MSH6 germline mutations in early-onset colorectal cancer patients without family history of the disease," British Journal of Cancer, vol. 95, no. 6, pp. 752756, 2006.

[36] M. D. Giráldez, F. Balaguer, L. Bujanda et al., "MSH6 and MUTYH deficiency is a frequent event in early-onset colorectal cancer," Clinical Cancer Research, vol. 16, no. 22, pp. 5402-5413, 2010.
[37] M. D. Giráldez, S. Castellví-Bel, F. Balaguer, V. Gonzalo, T. Ocaña, and A. Castells, "Lynch syndrome in colorectal cancer patients," Expert Review of Anticancer Therapy, vol. 8, no. 4, pp. 573-583, 2008.

[38] D. J. Ahnen, S. W. Wade, W. F. Jones et al., "The increasing incidence of young-onset colorectal cancer: a call to action," Mayo Clinic Proceedings, vol. 89, no. 2, pp. 216-224, 2014.

[39] M. Antelo, F. Balaguer, J. Shia et al., "A high degree of LINE1 hypomethylation is a unique feature of early-onset colorectal cancer," PLoS ONE, vol. 7, no. 9, Article ID e45357, 2012.

[40] J. Brändstedt, S. Wangefjord, B. Nodin et al., "Associations of anthropometric factors with KRAS and BRAF mutation status of primary colorectal cancer in men and women: a cohort study," PLoS ONE, vol. 9, no. 6, Article ID e98964, 2014.

[41] A. Goel, T. Nagasaka, C. N. Arnold et al., "The CpG island methylator phenotype and chromosomal instability are inversely correlated in sporadic colorectal cancer," Gastroenterology, vol. 132, no. 1, pp. 127-138, 2007.

[42] S. Ogino, T. Kawasaki, G. J. Kirkner, P. Kraft, M. Loda, and C. S. Fuchs, "Evaluation of markers for CpG Island Methylator Phenotype (CIMP) in colorectal cancer by a large populationbased sample," Journal of Molecular Diagnostics, vol. 9, no. 3, pp. 305-314, 2007.

[43] J. R. Jass, "Classification of colorectal cancer based on correlation of clinical, morphological and molecular features," Histopathology, vol. 50, no. 1, pp. 113-130, 2007.

[44] H. M. Müller, M. Oberwalder, H. Fiegl et al., "Methylation changes in faecal DNA: a marker for colorectal cancer screening?" The Lancet, vol. 363, no. 9417, pp. 1283-1285, 2004.

[45] J.-P. J. Issa, Y. L. Ottaviano, P. Celano, S. R. Hamilton, N. E. Davidson, and S. B. Baylin, "Methylation of the oestrogen receptor $\mathrm{CpG}$ island links ageing and neoplasia in human colon," Nature Genetics, vol. 7, no. 4, pp. 536-540, 1994.

[46] D. M. E. I. Hellebrekers, M. H. F. M. Lentjes, S. M. van den Bosch et al., "GATA4 and GATA5 are potential tumor suppressors and biomarkers in colorectal cancer," Clinical Cancer Research, vol. 15, no. 12, pp. 3990-3997, 2009.

[47] X.-L. Xu, J. Yu, H.-Y. Zhang et al., "Methylation profile of the promoter $\mathrm{CpG}$ islands of 31 genes that may contribute to colorectal carcinogenesis," World Journal of Gastroenterology, vol. 10, no. 23, pp. 3441-3454, 2004.

[48] M. O. Hiltunen, J. Koistinaho, L. Alhonen et al., "Hypermethylation of the WT1 and calcitonin gene promoter regions at chromosome 11P in human colorectal cancer," British Journal of Cancer, vol. 76, no. 9, pp. 1124-1130, 1997.

[49] Y. Baba, K. Nosho, K. Shima et al., "Hypomethylation of the IGF2 DMR in colorectal tumors, detected by bisulfite pyrosequencing, is associated with poor prognosis," Gastroenterology, vol. 139, no. 6, pp. 1855-1864, 2010.

[50] E. M. Wolff, H.-M. Byun, H. F. Han et al., "Hypomethylation of a LINE-1 promoter activates an alternate transcript of the MET oncogene in bladders with cancer," PLoS Genetics, vol. 6, no. 4, Article ID e1000917, 2010.

[51] P. A. Jones and S. B. Baylin, "The epigenomics of cancer," Cell, vol. 128, no. 4, pp. 683-692, 2007.

[52] E. Sunami, M. de Maat, A. Vu, R. R. Turner, and D. S. B. Hoon, "LINE-1 hypomethylation during primary colon cancer progression," PLoS ONE, vol. 6, no. 4, Article ID e18884, 2011. 


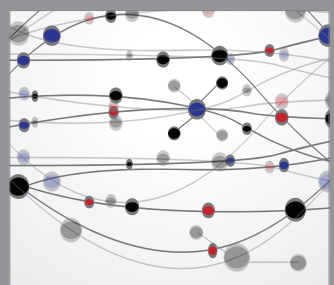

The Scientific World Journal
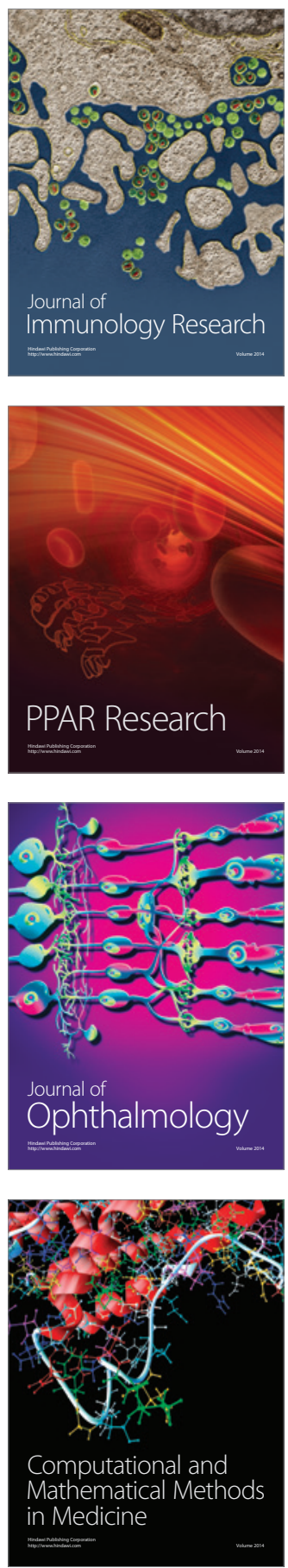

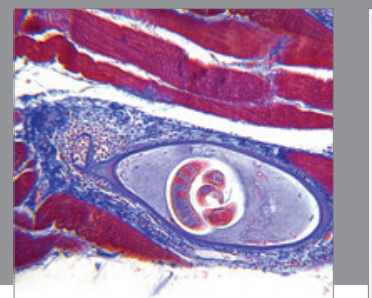

Gastroenterology

Research and Practice
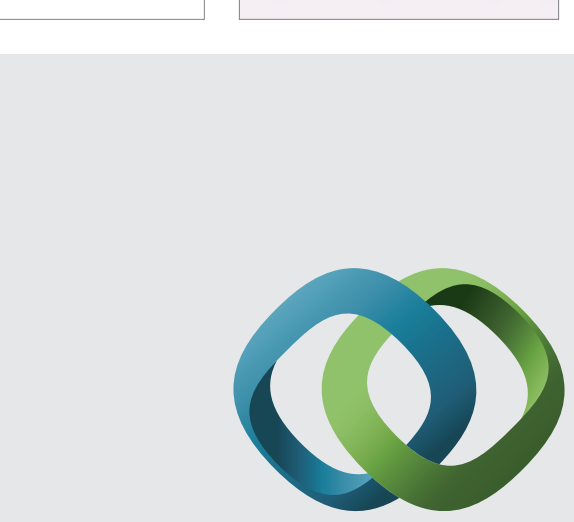

\section{Hindawi}

Submit your manuscripts at

http://www.hindawi.com
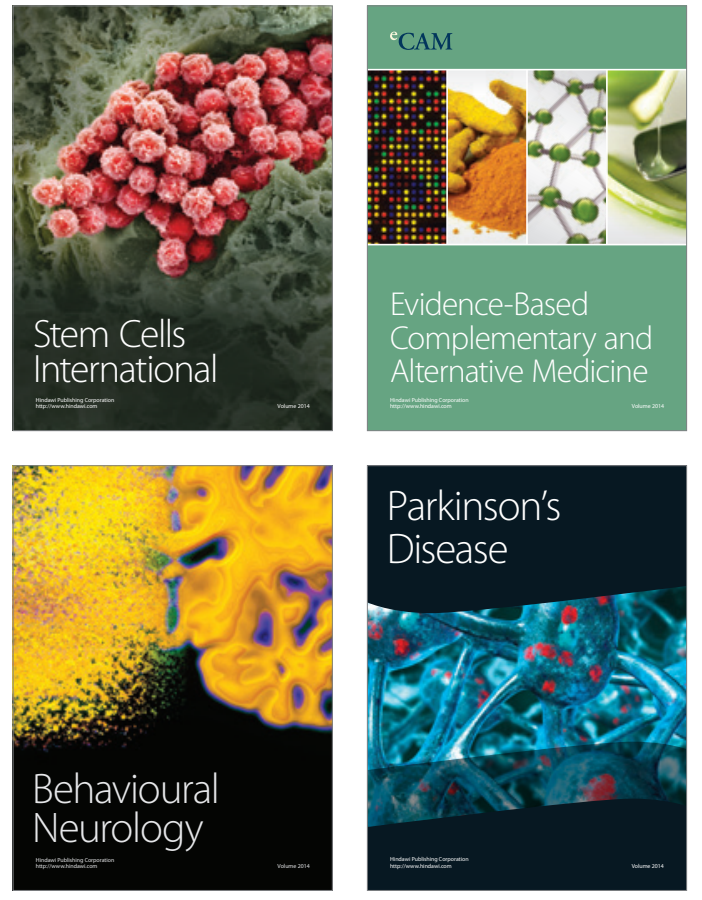
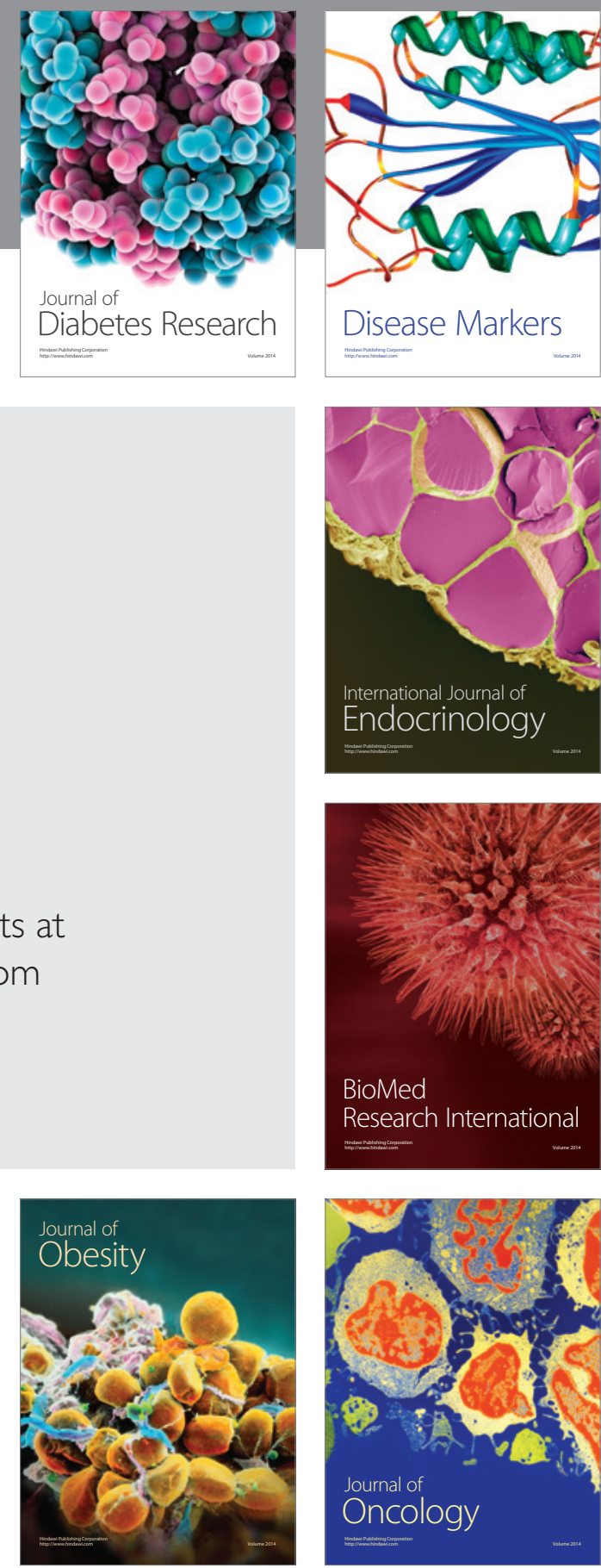

Disease Markers
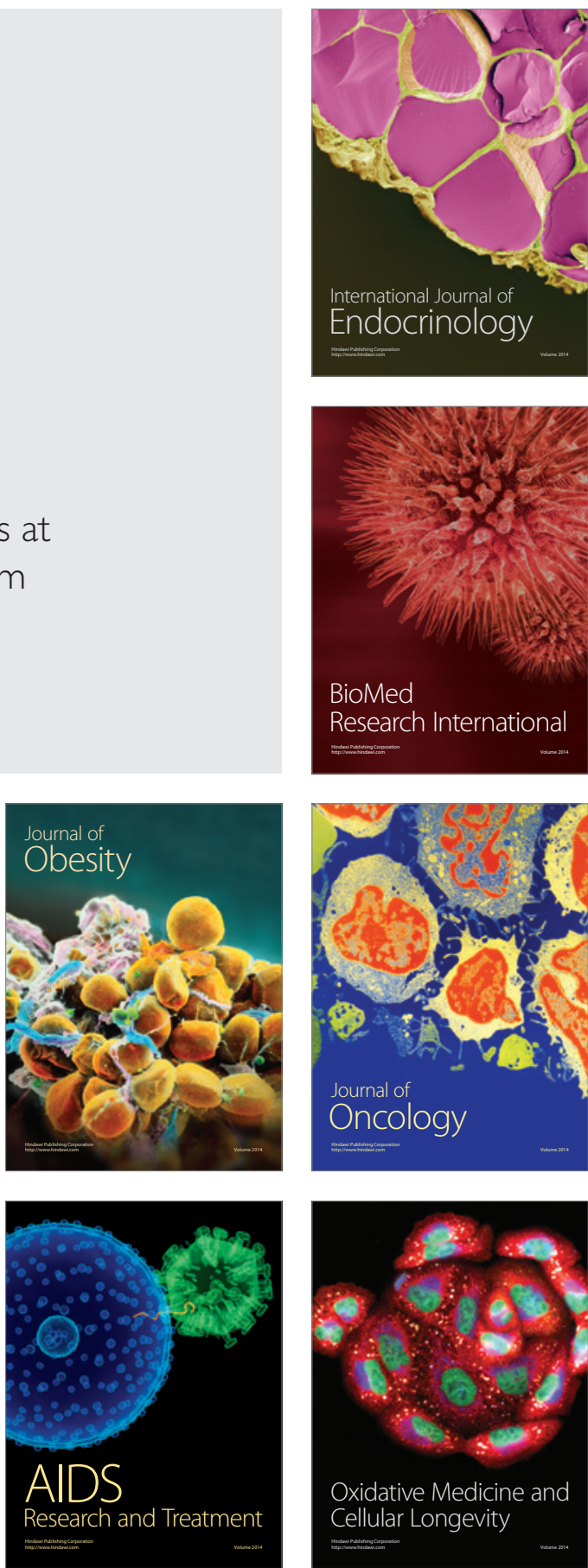